Pacific Journal of Mathematics

L-ORTHOGONALLY SCATTERED MEASURES

KONDAGUNTA SUNDARESAN AND WOJBOR WOYCZYNSK 


\title{
L-ORTHOGONALLY SCATTERED MEASURES
}

\author{
K. SUNDARESAN AND W. A. WoyCZYNSKI
}

Let $(X, \Sigma)$ be a measurable space and $H$ be a Hilbert space. Let $\mu$ be a measure on $\Sigma$ with values in $H$ such that $\mu(A)$ is orthogonal to $\mu(B)$ if $A, B$ are disjoint sets in $\Sigma$. Such measures are called orthogonally scattered measures and have been extensively studied during the past two decades by several authors. In this paper, the concept of lattice orthogonally scattered measures is introduced, this being a natural analogue of orthogonally scattered measures, when the measure $\mu$ takes values in a topological vector lattice. The main purpose of this paper is to study (1) Hahn extension, (2) Representation and (3) Radon-Nikodym theorem of lattice orthogonally scattered measures.

1. Introduction. In spite of many questions yet to be answered the theory of vector measures (i.e. measures taking values in a Banach space) seems to be a well-developed discipline today, Dinculeanu [1]. In particular when the range of the measure is a Hilbert space and the measure is orthogonally scattered (i.e. has orthogonal values on disjoint sets) the theory is especially deep and elegant and its origins go as far back as the early Wiener paper [13]. As highlights of this theory we mention the description of the space of real functions that are integrable with respect to such a measure and the applications of harmonic analysis of such measures to stationary Stochastic processes in the Wiener-Kolmogorov prediction theory.

Unlike his predecessors, recently Masani [6] (cf. also [7]) wrote an expository paper on the subject without using any probabilistic terminology. Generalizations of this theory were pursued in several directions by a number of authors. All of them, to the best of our knowledge, were trying to replace the Hilbert space in the range of the measure by a more general space (not necessarily even locally convex) while retaining the property of "independent" scatteredness of values of measure on disjoint sets. For example, the "independent" scatteredness may mean orthogonality of ranges of operators when the values of the measure are hermitian operators in a Hilbert space, and it means stochastic independence in the case of general random measures considered in Urbanik [12]. When the range space is a topological vector lattice, we have a natural concept of orthogonality namely the lattice theoretic one. In the present paper, we are making an attempt to study vector-valued measures taking values in certain topological vector lattices interpreting "independent" scatteredness in terms of lattice theoretic orthogonality ( $l$-orthogonally scattered mea- 
sures). The elegance of the Wiener-Kolmogorov theory stems from the fact that the positive measure associated with the Hilbert-space valued orthogonally scattered measure is not only mutually absolutely continuous with respect to the vector measure in question but it is also algebraically closely connected with it, [5]. In the case under consideration, we have a similar advantage.

It might be mentioned that there are several orthogonality concepts available in arbitrary Banach spaces. For an extensive discussion of such concepts we refer to James [4]. As pointed out in [4] the most interesting of these concepts is the following. (D) Let $B$ be a Banach space. If $x, y \in B, x$ is said to be orthogonal to $y$ if $\|x+\lambda y\| \geqq\|x\|$ for all real numbers $\lambda$. It has been shown recently in Sundaresan [11] that if $\operatorname{dim} B \geqq 2$ and if $F$ is a continuous function on $B \rightarrow R$ such that $F(x+y)=F(x)+F(y)$ whenever $x \perp y$ then $F$ is of the form $c\|x\|^{2}+l(x)(l(x))$ where $l \in B^{*}$ if $B$ is a Hilbert space (if $B$ is not isometric with a Hilbert space). It is for this reason that we have not considered measures orthogonally scattered where orthogonality is interpreted following the definition in (D).

In this paper we discuss the following three problems concerning l-orthogonally scattered measures:

(1) Hahn extension of these measures (§3).

(2) Representation theorem for such measures (\$4).

(3) Radon-Nikodym theorem for these measures (\$5).

Finally, we indicate some applications to random measures in the concluding $\S 6$. The problem in $\S 6$ is the essential motivation for the results discussed in $\S \S 3-5$.

The theory of o.s. measures, [6] and the theory of 1.o.s. measures have some similarities. When the range space is a Hilbert space $L_{2}(\mu)$, the theory of l.o.s. measure is a special case of o.s. measure. However, the 1.o.s. measure need not take values in a Hilbert space. The theories are independent of each other in general.

2. In this section, we state the notation, a few definitions, and elementary facts which are required in the subsequent sections.

If $(\mathfrak{X}, \wedge, \vee)$ is a vector lattice and $x, y \in \mathfrak{X}$ then $x$ is said to be orthogonal to $y$ in the lattice theoretic sense, in short $x \perp y$, if $|x| \wedge$ $|y|=0$. For basic definitions and properties of a vector lattice, see $\S \S 2$ and 3 in Chapter XII, Yosida [14]. In what follows, $\mathfrak{X}$ is a vector lattice of equivalence classes of measurable real-valued functions on a measure space $(\Omega, \Sigma, \mu)$, where $\Omega$ is a set, $\Sigma$ is a $\sigma$-algebra of subsets of $\Omega$, and $\mu$ is a $\sigma$-finite extended real-valued positive measure. We adopt here the measure theoretic terminology in Halmos [2]. A functional (nonlinear in general) $F: \mathfrak{X} \rightarrow R$ is said to be additive if $F(x+y)=F(x)+F(y)$ if $x \perp y$. If $\mathfrak{X}$ is a topological vector lattice, 
we require $F$ to be continuous. An additive functional $\rho$ on a vector lattice is said to be a modular if (1) $x=0 \Leftrightarrow \rho(x)=0$, (2) $|x| \leqq|y| \Rightarrow$ $\rho(x) \leqq \rho(y)$, (3) $0 \leqq x_{n} \uparrow x, \sup \rho\left(x_{n}\right)<\infty \Rightarrow x \in \mathfrak{X}$, and $\rho(x)=\sup \rho\left(x_{n}\right)$. $A$ vector lattice equipped with a modular is called a modular space. For a discussion of modular spaces, we refer to Matuszewska and Orlicz [8]. Condition (3) implies the axiom $C$ of completeness in [8].

A function $\|\cdot\|$ on a vector space into nonnegative real numbers is called an $F$-norm if (a) $\|x\|=0 \Leftrightarrow x=0$, (b) $\|-x\|=\|x\|$, and (c) $\|x+y\| \leqq\|x\|+\|y\|$ for all vectors $x$ and $y$. An $F$-space is a vector space, $E$, equipped with a $F$-norm $\|\cdot\|$ such that the metric space $(E, d)$, where $d(x, y)=\|x-y\|$, is complete.

If $(\mathfrak{X}, \rho)$ is a modular space then the function

$$
\|x\|_{\rho} \stackrel{\text { def }}{=} \inf \left\{\alpha \mid \rho\left(\frac{x}{\alpha}\right) \leqq \alpha, \alpha>0\right\}
$$

is an $F$-norm on $\mathfrak{X}$ rendering $\mathfrak{X}$ an $F$-space. The $F$-norm $\|\cdot\|_{\rho}$, induced by the modular $\rho$, is said to be absolutely continuous if $\rho\left(x_{n}\right) \rightarrow 0 \Rightarrow$ $\left\|x_{n}\right\|_{\rho} \rightarrow 0$. In what follows, we always assume that the $F$-norm induced by the modular under consideration is absolutely continuous. For a discussion of the absolute continuity of $\|\cdot\|_{\rho}$, we refer to [8].

$\mathscr{B}_{0}$ stands for a pre-ring of subsets of a set $T$ i.e. (1) $A, B \in \mathscr{B}_{0} \Rightarrow$ $A \cap B \in \mathscr{B}_{0}$, (2) $A, B \in \mathscr{B}_{0}, A \subset B$ there is a finite increasing sub-family $C_{1}, \cdots, C_{n} \in \mathscr{B}_{0}$ such that $A=C_{1}, B=C_{n}$ and $C_{i} \sim C_{i-1} \in \mathscr{B}_{0}, i=1, \cdots, n$. We denote the $\sigma$-ring generated by $\mathscr{B}_{0}$ by $\mathscr{B}$.

Definition. (a) A mapping $\xi: \beta_{0} \rightarrow \mathfrak{X}$ is said to be a $l$-orthogonally scattered (l.o.s.) measure if $\xi(A \cup B)=\xi(A)+\xi(B)$, and $\xi(A) \perp \xi(B)$ whenever $A \cap B=\varnothing, A, B \in \mathscr{B}_{0}$, and $A \cup B \in \mathscr{B}_{0}$.

(b) If $\mathfrak{X}$ is a topological vector lattice then $\xi: \mathscr{B}_{0} \rightarrow \mathfrak{X}$ is said to be a countably additive l-orthogonally scattered (c.a.l.o.s.) measure if $\xi$ is a 1.o.s. measure and in addition $\xi\left(\bigcup_{i=1}^{\infty} A_{i}\right)=\sum_{i=1}^{\infty} \xi\left(A_{i}\right)$ whenever $\left\{A_{i}\right\}$ is a pairwise disjoint sequence from $\mathscr{B}_{0}$ such that $\bigcup_{i=1}^{\infty} A_{i} \in \mathscr{B}_{0}$.

EXAMPLE. (Indicator c.a.l.o.s. measure.) Let $T=\Omega, \mathscr{B}=\Sigma$ and $f \in \mathfrak{X}$ where $\mathfrak{X}$ is normal (i.e. if $g \in \mathfrak{X}, g^{\prime}$ is a nonnegative measurable function such that $0 \leqq g^{\prime} \leqq g$ then $\left.g^{\prime} \in \mathfrak{X}\right)$. Then $\xi^{f}$ defined by the formula $\xi^{f}(A)=f \cdot \mathfrak{X}_{A}$ is a c.a.l.o.s. measure on $\Sigma$ into $\mathfrak{X}$.

If $\xi$ is a c.a.l.o.s. measure on $\mathscr{B}_{0}$ with values in a topological vector lattice $\mathfrak{X}$ and $F$ is an additive functional on $\mathfrak{X}$ then, $\xi_{F}=F \circ \xi$ is a countably additive real measure on $\mathscr{P}_{0}$. We collect here few elementary facts concerning $\xi$ and $\xi_{F}$. Since the results are immediate consequences of the definitions, the proofs are not supplied. 
(1) If $A, B \in \mathscr{B}_{0}, B \sim A \in \mathscr{B}_{0}$ and $A \sim B \in \mathscr{B}_{0}$, then

$$
F(\xi(A)-\xi(B))=F(\xi(A \sim B))+F(-\xi(B \sim A)) .
$$

(2) If $A, B \in \mathscr{B}_{0}, B \subset A, A \sim B \in \mathscr{B}_{0}$, then

$$
F(\xi(A)-\xi(B))=F(\xi(A))-F(\xi(B)) \cdot
$$

(3) If $A, B \in \mathscr{B}_{0}$ and $A \sim B \in \mathscr{B}_{0}$, then

$$
\xi(A \sim B)=\xi(A)-\xi(A \cap B) \text {. }
$$

(4) If $A, B \in \mathscr{B}_{0}, A \sim B \in \mathscr{B}_{0}, B \sim A \in \mathscr{B}_{0}$, then

$$
\xi(A \Delta B)=\xi(A)+\xi(B)-2 \xi(A \cap B) \text { if } A \Delta B \in \mathscr{S}_{0} \text {. }
$$

Further, we have the following elementary lemma.

Lemma 1. Suppose $\xi: \mathscr{B}_{0} \rightarrow \mathfrak{X}$ is a c.a.l.o.s. measure and $F: \mathfrak{X} \rightarrow R$ is an additive functional. Then the following two statements are equivalent.

(a) For all $A, B \in \mathscr{B}_{0}$ such that $B \subseteq A$

$$
F(\xi(A)-\xi(B))=\nu(A)-\nu(B)
$$

where $\nu: \mathscr{B}_{0} \rightarrow R$ is a c.a. measure.

(b) $\xi_{F^{\prime}}=\nu$.

3. Here we proceed to discuss the Hahn extension of c.a.l.o.s. measure defined on a pre-ring $\mathscr{B}_{0}$. In this connection we recall the classical Hahn extension theorem on p. 54 in Halmos [2]. For convenience if $\nu$ is any real countable additive measure on $\mathscr{B}_{0}$ then its Hahn extension to $\mathscr{B}$ is denoted by $\bar{\nu}$.

THEOREM 1. Let $\xi: \mathscr{B}_{0} \rightarrow \mathfrak{X}$ be a c.a.l.o.s. measure where $(\mathfrak{X}, \rho)$ is a modular space and $\widetilde{\xi}_{\rho}$ is the Hahn extension of $\xi_{\rho}$ to $\mathscr{B}$. Let $\mathscr{F}=\left\{A \mid A \in \mathscr{B}, \xi_{\rho}(A)<\infty\right\}$. Then there exists a unique extension $\tilde{\xi}$ of $\xi$ to $\mathscr{F}$ such that $(\tilde{\xi})_{\rho}=\tilde{\xi}_{\rho+}$.

Proof. Let $\mathscr{R}$ be the ring generated by $\mathscr{B}_{0}$. Let $\hat{\xi}$ be an extension of $\xi$ to $\mathscr{R}$. The existence of such an extension is verified as follows. If $A \in \mathscr{R}$, choose an arbitrary family of pairwise disjoint sets $A_{1}, \cdots, A_{n} \in \mathscr{\mathscr { O }}$ such that $A=\bigcup_{i=1}^{n} A_{i}$ and let $\hat{\xi}(A)=\sum_{i=1}^{n} \xi\left(A_{i}\right)$. It is verified that $\hat{\xi}$ is a c.a.l.o.s. measure on $\mathscr{R}$ into $\mathfrak{X}$ and an extension of $\xi$. By Theorem D, p. 56 of [2], it follows that if $A \in \mathscr{F}$, $\varepsilon>0$, there exists $B \in \mathscr{R}$ such that $\tilde{\xi}_{\rho}(A \Delta B)<\varepsilon$. Thus there exists a sequence $\left\{B_{n}\right\} \subset \mathscr{R}$ such that $\widetilde{\xi}_{\rho}\left(A \Delta B_{n}\right) \rightarrow 0$ as $n \rightarrow \infty$. Further it is verified that $\tilde{\xi}_{\rho}\left(B_{m} \Delta B_{n}\right) \rightarrow 0$ as $n \rightarrow \infty$. Since 


$$
\begin{aligned}
\tilde{\xi}_{\rho}\left(B_{m} \Delta B_{n}\right) & =\rho\left(\xi\left(B_{m} \sim B_{n}\right)+\xi\left(B_{n} \sim B_{m}\right)\right) \\
& \left.=\rho\left(\xi\left(B_{m} \sim B_{n}\right)\right)+\rho\left(\xi B_{n} \sim B_{m}\right)\right),
\end{aligned}
$$

it follows that

$$
\rho\left(\xi\left(B_{m} \sim B_{n}\right)\right) \rightarrow 0 \text { and } \rho\left(\xi\left(B_{n} \sim B_{m}\right)\right) \rightarrow 0 .
$$

Hence, from (1) in $\S 2$

$$
\rho\left(\xi\left(B_{m}\right)-\xi\left(B_{n}\right)\right) \rightarrow 0 .
$$

Since $\rho$ is absolutely continuous

$$
\left\|\xi\left(B_{m}\right)-\xi\left(B_{n}\right)\right\| \rightarrow 0 .
$$

Since $\mathfrak{X}$ is complete, there exists an $x \in \mathfrak{X}$ such that $\xi\left(B_{n}\right) \rightarrow x$. If not $\left\{C_{n}\right\} \subset \mathscr{F}$ is another sequence such that $\tilde{\xi}_{0}\left(A \Delta C_{n}\right) \rightarrow 0$ then $\tilde{\xi}\left(C_{n}\right) \rightarrow y$ for some $y \in \mathfrak{X}$. Since $\tilde{\xi}_{\rho}\left(B_{n} \Delta C_{n}\right) \rightarrow 0, \rho\left(\xi\left(B_{n}\right)-\xi\left(C_{n}\right)\right) \rightarrow 0$. Hence $\left\|\xi\left(B_{n}\right)-\xi\left(C_{n}\right)\right\| \rightarrow 0$. Thus $x=y$. Let $\tilde{\xi}: \mathscr{F} \rightarrow \mathfrak{X}$ be the function defined by $\tilde{\xi}(A)=\lim \tilde{\xi}\left(A_{n}\right)$, if $\left\{A_{n}\right\} \subset \mathscr{R}$ is a sequence such that $\tilde{\xi}_{\rho}\left(A \Delta A_{n}\right) \rightarrow 0$. Indeed $\tilde{\xi}$, thus defined, is a c.a.l.o.s. measure on $\mathscr{F}$. For let $A, B \in \mathscr{F}, A \cap B=\varnothing$. Let $\left\{A_{n}\right\},\left\{B_{n}\right\}$ be sequences in $\mathscr{R}$ such that $\widetilde{\xi}_{\rho}\left(A \Delta A_{n}\right) \rightarrow 0$, and $\widetilde{\xi}_{\rho}\left(B \Delta B_{n}\right) \rightarrow 0$. Arguing as in Theorem A, page 168, Halmos [2], it is verified that $\xi_{\rho}\left(\left(A_{n} \sim B_{n}\right) \triangle A\right) \rightarrow 0$, and $\widetilde{\xi}_{\rho}\left(\left(B_{n} \sim A_{n}\right) \Delta B\right) \rightarrow 0$. Hence $\tilde{\xi}\left(A_{n} \sim B_{n}\right) \rightarrow \tilde{\xi}(A)$, and $\tilde{\xi}\left(B_{n} \sim A_{n}\right) \rightarrow \tilde{\xi}(B)$. Arguing as in the proof of Lemma 1 in [8], it follows that there exists a subsequence $\left\{n_{i}\right\}$ of integers such that $\tilde{\xi}\left(A_{n_{i}} \sim B_{n_{i}}\right) \rightarrow \tilde{\xi}(A)$ and $\tilde{\xi}\left(B_{n_{i}} \sim A_{n_{i}}\right) \rightarrow \tilde{\xi}(B), \mu$ a.e. Since $\tilde{\xi}\left(A_{n_{i}} \sim B_{n_{i}}\right) \perp \tilde{\xi}\left(B_{n_{i}} \sim A_{n_{i}}\right)$ it follows that $\tilde{\xi}(A) \perp \tilde{\xi}(B)$. Further $\tilde{\xi}(A \cup B)=\lim \tilde{\xi}\left(\left(A_{n} \sim B_{n}\right) \cup\left(B_{n} \sim\right.\right.$ $\left.\left.A_{n}\right)\right)=\tilde{\xi}(A)+\widetilde{\xi}(B)$. From the definition of $\tilde{\xi}$, it is verified that $\tilde{\xi}$ is $\widetilde{\xi}_{\rho}$-absolutely continuous. Thus from the preceding it follows that $\tilde{\xi}$ is a c.a.l.o.s. measure, since $\tilde{\xi}_{\rho}$ is countably additive. An easy application of Lemma 1 yields $\left(\bar{\xi}_{\rho}\right)=\bar{\xi}_{\rho} \mid \mathscr{F}$.

We proceed to exhibit a counterexample to show that in general a c.a.l.o.s. measure does not admit a Hahn extension with respect to arbitrary additive functionals on $\mathfrak{X}$.

EXAMPLE. Let $\mathscr{R}$ be the ring of all finite unions of disjoint bounded left-closed right-open intervals in the real line $R$. Let $\mathfrak{X}=$ $L_{1}(\mu)$ where $\mu$ is the Lebesgue measure.

$\mathfrak{X}$ is a vector lattice under the natural partial ordering $x \leqq y$ whenever $x(t) \leqq y(t)$ for $\mu$ almost all $t \in R$. Let $\varphi: R \rightarrow R$ be a continuous function such that $\varphi$ has support in $[1 / 2,1], \varphi$ not identically 0 , and range $\varphi \subset[0,1]$. Let $F$ be the additive functional defined by

$$
F(x)=\int_{R} \varphi(x(t)) d \mu(t), \quad x \in \mathfrak{X} .
$$


$F$ is well defined since $\varphi \circ x$ is a bounded measurable function with bounded support. Let $\xi: \mathscr{R} \rightarrow L_{1}(\mu)$ be a c.a.1.o.s. measure defined by

$$
\xi(A)=\sum_{1}^{\infty} \frac{1}{n} \chi_{A \cap I_{n}},
$$

where $I_{n}=[n, n+1)$.

It is verified that if $\bar{\xi}_{F}$ is the extension of $\xi_{F}$ to $\sigma(\mathscr{R})$, then $\bar{\xi}_{F}(R)<\infty$, indeed $\bar{\xi}_{F}(R)=\lim \bar{\xi}_{F}[0, n)=\xi_{F}[0,2)$. If $\xi$ has an extension $\bar{\xi}$ to $\mathscr{F}=\left\{A \mid A \subset R, \bar{\xi}_{F}(A)<\infty\right\}$, then since $\bar{\xi}_{F}\left(R^{+}\right)<\infty, \bar{\xi}\left(R^{+}\right)$ is defined. However, since $\bar{\xi}$ is c.a.l.o.s. measure

$$
\bar{\xi}\left(R^{+}\right)=\sum_{n \geq 1} \bar{\xi}\left(I_{n}\right)=\sum \frac{1}{n} \chi_{I_{n}}
$$

which is not in $L_{1}(\mu)$, a contradiction.

4. In the present section we deal with certain structure theorems for c.a.l.o.s. measures. We recall briefly the necessary terminology. $(\Omega, \Sigma, \mu)$ is a measure space where $\Sigma$ is a $\sigma$-algebra of subsets of $\Omega$ and $\mu$ is an extended real-valued nonnegative measure. If $\mathscr{\mathscr { H }}_{i}$ are two $\sigma$-rings of sets then a mapping $\tau: \mathscr{B}_{1} \rightarrow \mathscr{\mathscr { B } _ { 2 }}$ is said to be a $\sigma$ homomorphism if $A_{i} \in \mathscr{B}_{1}, i \geqq 1$ implies $\tau\left(\bigcup_{i \geqq 1} A_{i}\right)=\cup \tau\left(A_{i}\right)$ and $\tau\left(A_{1} \sim A_{2}\right)=\tau\left(A_{1}\right) \sim \tau\left(A_{2}\right)$.

Proposition 1. Let $\mathfrak{X}$ be an $F$-space of equivalence classes of measurable real-valued functions on a measure space $(\Omega, \Sigma, \mu), \mu$ not necessarily $\sigma$-finite and let $\xi: \mathscr{B} \rightarrow \mathfrak{X}$ be a c.a.l.o.s. measure on a $\sigma$ ring $\mathscr{B}\left(\subset 2^{T}\right)$. Then there exists a function $f \in \mathfrak{X}$ and a $\sigma$-homomorphism $h^{\Delta}: \mathscr{B} \rightarrow \Sigma / \Delta$ such that

$$
\xi(A)=f \chi_{h \Lambda_{(A)}}, \quad A \in \mathscr{B} .
$$

Proof. We note that if $\mathscr{A} \subset \mathscr{B}$ such that $A_{1}, A_{2} \in \mathscr{A}$ implies $A_{1} \cap A_{2}=\varnothing$ and $A \in \mathscr{A}$ implies $\xi(A) \neq 0$ then card $\mathscr{A} \leqq \boldsymbol{\aleph}_{0}$. For if card $\mathscr{A}>\boldsymbol{\aleph}_{0}$ consider $\{\|\xi(A)\| A \in \mathscr{A}\}$. There exists $\mathscr{A}_{0} \subset \mathscr{A}$ card $\mathscr{A}_{0}=\boldsymbol{\aleph}_{0}$ and a positive number $\alpha$ such that $A \in \mathscr{A}_{0}$ implies $\|\xi(A)\|>\alpha$. In such a case, since $\xi$ is an l.o.s. measure it is at once verified that $\left\{\sum_{i=1} \xi\left(A_{i}\right) \mid A_{i} \in \mathscr{A}_{0}\right\}_{n \geqq 1}$ is not a Cauchy sequence. Hence it does not converge to $\xi\left(\cup A_{i}\right)$, a contradiction.

Now applying Zorn's lemma it follows that there exists a maximal family $\mathscr{A}_{0} \subset \mathscr{B}$ such that $\mathscr{A}_{0}$ is pairwise disjoint and $A \in \mathscr{A}_{0}$ implies $\xi(A) \neq 0$. Let $T_{0}=\bigcup_{A \in \mathscr{S}_{0}} A$ and $\xi\left(T_{0}\right)=f \in \mathfrak{X}$. Let $h^{4}: \mathscr{B} \rightarrow \Sigma / \Delta$ be the mapping defined by $h^{\Delta}(A)=\sup \xi(A), A \in \mathscr{B}$. The mapping $h^{4}$ is a $\sigma$-homomorphism on $\mathscr{B}$ into $\Sigma / \Delta$. Indeed 


$$
\begin{aligned}
h^{\Delta}(A \cup B) & =\sup (\xi(A \sim B)+\xi(A \cap B)+\xi(B \sim A)) \\
& =\sup (\xi(A \sim B)+\xi(A \cap B)) \cup \sup (\xi(A \cap B)+\xi(B \sim A)) \\
& =h^{\Delta}(A) \cup h^{\Delta}(B)
\end{aligned}
$$

and

if $A \subset B$ then

$$
h^{\Delta}(B \sim A)=h^{\Delta}(B) \sim h^{\Delta}(A)
$$

since $h^{\Delta}(B)=h^{\Delta}(B \sim A) \cup h^{\Delta}(A)$ and $h^{\Delta}(A)$ and $h^{\Delta}(B \sim A)$ are disjoint. The fact that $h^{4}$ is a $\sigma$-homomorphism is implied by the $\sigma$-additivity and $l$-orthogonal scatteredness of $\xi$. Now if $A \subset T_{0}$ (the case $A \not \subset T_{0}$ may be reduced to the latter because $\xi\left(A \sim T_{0}\right)=0$ ) then $T_{0}=A \cup$ $\left(T_{0} \sim A\right)$ and

$$
f=\xi\left(T_{0}\right)=\xi(A)+\xi\left(T_{0} \sim A\right) .
$$

Hence

$$
f=f \chi_{h^{\Delta}(A)}+f \chi_{h^{\Delta}\left(T_{0} \sim A\right)} .
$$

Since $\xi(A) \perp \xi\left(T_{0} \sim A\right)$ and support $f \chi_{h^{\Delta}(A)}=h_{\Delta}(A)=$ support $\xi(A)$ we obtain $\xi(A)=f \chi_{h A_{(A)}}$, completing the proof of the proposition.

A $\sigma$-algebra of sets is said to be $\sigma$-perfect if every $\sigma$-filter is determined by a point. We recall that a sub-family $\mathscr{F}$ of a $\sigma$-algebra $\Sigma$ of subsets of a set $\Omega$ is said to be a $\sigma$-filter if (a) $A \in \mathscr{F}, B \supset A$, $B \in \Sigma$ implies $B \in \mathscr{F}$, and (b) $A_{i} \in \mathscr{F}, i \geqq 1$ implies $\cap A_{i} \in \mathscr{F}$. A filter $\mathscr{F}$ of sets in $\Sigma$ is said to be determined by a point $x$ if $\mathscr{F}=$ $\{A \mid A \in \Sigma, x \in A\}$. Further we note that every $\sigma$-algebra of sets is isomorphic to a $\sigma$-perfect $\sigma$-algebra of sets, Sikorski [10]. We proceed to show that Proposition 1 concerning the structure of $\xi$ could be improved in certain special cases. We adopt the following notation: $\mathscr{L}_{\infty}(\Omega, \Sigma, \mu)$ is the space of all bounded measurable functions $\tilde{f}$ on $(\Omega, \Sigma, \mu)$ and $L_{\infty}(\Omega, \Sigma, \mu)$ is the space of all equivalence classes of $\mathscr{L}_{\infty}(\Omega, \Sigma, \mu)$. In the next proposition we choose for $\mathfrak{X}=L_{\infty}(\Omega, \Sigma, \mu)$ with $\mathscr{L}_{\infty} \ni \tilde{f} \rightarrow(\widetilde{f})^{4} \in L_{\infty}$ as the canonical mapping.

Proposition 2. If $\mu$ is $\sigma$-finite, $\xi: \mathscr{B} \rightarrow L_{\infty}(\Omega, \Sigma, \mu)$ is an c.a.l.o.s. measure on a $\sigma$-perfect $\sigma$-algebra $\mathscr{B}$ of subsets of $T$ then the homomorphism $h^{\Delta}$ of the preceding proposition is induced by a pointwise mapping, i.e. there exists a mapping $\tau: \Omega \rightarrow T$ and there exists an $f \in \mathscr{L}_{\infty}(\Omega, \Sigma, \mu)$ such that

$$
\xi(A)=\left(\tilde{f} \tilde{\chi}_{\tau^{-1}(A)}\right)^{4}, \quad A \in \mathscr{B} .
$$

Proof. From the hypothesis on $\mu$ (causing its strict localizability) 
there exists a lifting on $L_{\infty}$ i.e. a multiplicative linear mapping $\lambda: L_{\infty} \rightarrow \mathscr{L}_{\infty}$ such that (a) $(\lambda(f))^{d}=f$, (b) $\lambda(1)=1$, (c) $f \geqq 0 \Rightarrow \lambda(f) \geqq 0$. (Chapter 4, Ionescu-Tulcea [3].) It follows that $\lambda$ is continuous and it is a vector lattice homomorphism. Hence $\lambda \circ \xi: \mathscr{B} \rightarrow \mathscr{L}_{\infty}$ is a c.a.l.o.s. measure. Now we proceed as in the proof of the previous proposition. Let us define $h: \mathscr{B} \rightarrow \Sigma$ by setting $h(A)=\operatorname{support} \lambda(\xi(A))$ and as before it is verified that $h$ is a $\sigma$-homomorphism of $\mathscr{B}$ into $\Sigma$. Since $\mathscr{B}$ is $\sigma$-perfect, every $\sigma$-homomorphism of $\mathscr{B}$ into any $\sigma$ algebra of sets is induced by some point-wise mapping [10]. Hence there is $\tau: \Omega \rightarrow T$ such that $h(A)=\tau^{-1}(A), A \in \mathscr{B}$. As in Proposition 1 , it is verified that $\lambda(\xi(A))=\widetilde{f} \widetilde{\chi}_{\tau^{-1}(A)}, A \in \mathscr{B}, \widetilde{f}$ being by definition equal to $\lambda(\xi(T))$ from which $(*)$ follows.

Since for $1 \leqq p<\infty$ there exists no positive linear lifting on $L_{p}$, the proof of Proposition 2 cannot be carried over for $L_{p}$-spaces. Hence, in general, we cannot claim that every c.a.l.o.s. measure is a composition of indicator c.a.l.o.s. measure and some rearrangement $(\tau)$ of the underlying set. However, if we restrict the class of $\sigma$-algebras $\mathscr{B}$ on which $\xi$ is defined, we can prove the similar result. For completeness sake, let us recall that the $\sigma$-algebra $\mathscr{B}$ is said to be an absolute Borel $\sigma$-algebra if it is a $\sigma$-algebra of Borel subsets of a topological space homeomorphic to a Borel subset of a Hilbert cube. For instance, every $\sigma$-algebra of Borel subsets of a separable complete metric space is absolutely Borel. See page 138 in [10].

Proposition 3. Let $\mathfrak{X}$ be an $F$-space of equivalence classes of realvalued measurable functions on a measurable space $(\Omega, \Sigma, \mu), \mu$ not necessarily $\sigma$-finite, and let $\xi: \mathscr{B} \rightarrow \mathfrak{X}$ be a c.a.l.o.s. measure on the $\sigma$-algebra $\mathscr{B}$ of subsets of $T$ where it is assumed that $\mathscr{B}$ is absolutely Borel. Then the $\sigma$-homomorphism $h^{4}$ of Proposition 1 is induced by a point-wise mapping i.e. there exists a function $f \in \mathfrak{X}$ and a mapping $\tau: \Omega \rightarrow T$ such that

$$
\xi(A)=f \cdot \chi_{:-1(A)}^{4}, \quad A \in \mathscr{B} .
$$

The proof of this proposition is very similar in details to the preceding one after noting Theorem 3.2.5, p. 139 [10]. Therefore, the details are omitted.

5. The real measure $\xi_{F}$ as defined in $\S 2$ is absolutely continuous with respect to the c.a.l.o.s. measure $\xi$, and in this section we discuss the existence of Radon-Nikodym derivative of $\xi$ with respect to $\xi_{F}$.

Theorem 2. Let $\xi: \mathscr{B} \rightarrow L_{p}(\Omega, \Sigma, \mu), 1 \leqq p \leqq \infty$, be an c..al.o.s. measure. Let $F$ be a fixed nonnegative additive functional on $L_{p}(\Omega, \Sigma, \mu)$. 
If there exists $A \in \mathscr{B}$ such that (1) $0<\xi_{A}(A)<\infty$, (2) $\left(A, \mathscr{B}_{A},|\xi|\right)$ is a non-atomic measure space, where $|\xi|$ is the total variation of $\xi$ and $\mathscr{B}_{A}$ is the trace of $\mathscr{B}$ on $A$ then $\xi$ does not admit a RadonNikodym derivative with respect to $\xi_{F}$.

Proof. We can assume that $\xi$ is absolutely continuous with respect to $\xi_{F}$, for otherwise $\xi$ does not admit a Radon-Nikodym derivative. Further, let $f$ be the function in $L_{p}(\Omega, \Sigma, \mu)$ determined by $\xi$ so that $\xi(A)=f \cdot \chi_{h_{(A)}(A)}, h^{\Delta}$ being the map defined in Proposition 1, $\S 4$. For the sake of simplicity and without loss of generality, we assume that $f \geqq 0$. Because of the representation of $\xi$ and since $\xi<<\xi_{F}$ it is verified that $|\xi|<<\xi_{F}$. Indeed, if $B \in \mathscr{B}$ and $\xi(B)=0$ and $|\xi|(B)=$ $\delta>0$, there exists $B_{1} \in \mathscr{B}$ such that $\left\|\xi\left(B_{1}\right)\right\|>0$. Hence $\xi_{F}\left(B_{1}\right) \neq 0$. Since $F$ is additive, $\xi\left(B_{1}\right) \neq 0$. Since $\xi(B)=0$ and $f \geqq 0, f \chi_{h} \Delta_{\left(B_{1}\right)}=$ $\xi\left(B_{1}\right)=0$. Further we note the following: (a) there exist $\alpha>0$, and $A_{1} \in \mathscr{B}, A_{1} \subset A$ such that $\xi\left(A_{1}\right) \neq 0$ and $f \chi_{h^{\Delta}\left(A_{1}\right)} \geqq \alpha \chi_{h^{\Delta}\left(A_{1}\right)}$, (b) the measure $\xi_{F}\left|\Sigma_{A_{1}}<<\mu h^{4}\right| \Sigma_{A_{1}}$. For if (a) were to be false then for each $\alpha>0$ and for every set $B \subset A, B \in \mathscr{B}$ such that $\xi(B) \neq 0$ there exists a set $B_{1}, B_{1} \in \mathscr{B}, B_{1} \subset B$ with $f \chi_{h^{4}\left(B_{1}\right)}<\alpha$. Pick $A_{1} \subset A$ such that $\xi\left(A_{1}\right) \neq 0$ and $f \chi_{h^{4}\left(A_{1}\right)}<\alpha$. Then either $\xi\left(A \sim A_{1}\right)=0$ or else repeating the above procedure we obtain $\xi\left(A_{2}\right) \neq 0$ and $f \chi_{h^{\Delta}\left(A_{2}\right)}<\alpha$. Thus we obtain a finite family of pairwise disjoint $\mathscr{B}$-measurable sets $\left\{A_{1}, A_{2}, \cdots, A_{n}\right\}$ such that $\xi\left(A \sim \bigcup_{i=1}^{n} A_{i}\right)=0$ and $f \chi_{h \Delta\left(\cup A_{i}\right)}<\alpha$ or else there exists an infinite sequence of pairwise disjoint $\mathscr{B}$-measurable sets $\left\{A_{i}\right\}_{i \geqq 1}, \xi\left(A_{i}\right) \neq 0$ such that $f \chi_{h^{\Delta}\left(\cup A_{i}\right)}<\alpha$. In the first case, $f \chi_{h^{4}(A)}<\alpha$. We shall show in the second case also the same holds. Let

$$
\Gamma=\left\{\left\{A_{i}\right\}_{i \geqq 1} \mid A_{i} \cap A_{j}=\varnothing, i \neq j, A_{i} \in \mathscr{B}, A_{1} \subset A \text { and } f \chi_{h^{\lrcorner}\left(A_{i}\right)}<\alpha\right\} .
$$

Partially order $\Gamma$ by inclusion. If $\mathscr{C}$ is a chain in $\Gamma$ and if $A, B \in \cup \mathscr{C}$ then $A \cap B=\varnothing$. Since $A \in \cup \mathscr{E}$ implies $\xi(A) \neq 0, \cup \mathscr{E}$ is countable. Further $A \in \cup \mathscr{E}$ implies $f \chi_{h^{\Delta}(A)}<\alpha$. Thus $\cup \mathscr{C}$ is a member of $\Gamma$. Hence by Zorn's lemma, there exists a maximal sequence $\mathscr{A}_{0}=$ $\left\{A_{i \geqq 1}\right\} \in \Gamma$. Now if $\xi\left(A \sim \bigcup_{i \geqq 1} A_{i}\right) \neq 0$, it is verified that the maximality of $\mathscr{A}_{0}$ is contradicted. Thus

$$
\xi(A)=\xi\left(\bigcup_{i \geqq 1} A_{i}\right)=f \chi_{h\lrcorner\left(\cup A_{i}\right)}<\alpha .
$$

i.e. $f \chi_{h \iota_{(A)}}<\alpha$. Thus if (a) is false for every $\alpha>0, f \chi_{h^{\Delta}(A)}<\alpha$. Since $f \geqq 0$ this implies that $\xi(A)=f \chi_{h^{4}(A)}=0$, contradicting $\xi_{F}(A)>0$. This completes the proof of (a) and there exists $A_{1} \in \mathscr{B}, A_{1} \subset A$, $\xi\left(A_{1}\right) \neq 0$ such that $f \chi_{h^{\Delta}\left(A_{1}\right)} \geqq \alpha \chi_{h^{4}\left(A_{1}\right)}$ for some positive number $\alpha$.

(b) follows directly from the representation of $\xi$ stated in Proposition 1. 
Since

$$
\|\xi(C)\|^{p}=\int f^{p} \chi_{h{ }^{4}(C)} d \mu<\infty
$$

for all $C \in \mathscr{B}_{1} C \subset A_{1}$ it is verified that $\mu \circ h^{4} \mid \Sigma_{A_{1}}$ is a finite measure. Since $\xi_{F} \mid \Sigma_{A_{1}}$ is a finite positive measure, we can chose a $\beta>0$ such that $\xi_{F}\left(A_{1}\right)-\beta \mu \circ h^{\Delta}\left(A_{1}\right)>0$. If for every $B \subset A_{1}, B \in \mathscr{B}, \xi_{F}(B)-$ $\beta \mu \circ h^{4}(B) \geqq 0$ then $\xi_{F}(B)=0$ implies $\mu \circ h^{4}(B)=0$ i.e. $\left(^{*}\right) \mu \circ h^{4} \mid \Sigma_{A_{1}}<<$ $\xi_{F} \mid \Sigma_{A_{1}}$. Thus either (**) there exists a set $A_{2} \subset A_{1}$ such that $0 \neq \nu=$ $\xi_{F}\left|\Sigma_{A_{2}}-\beta \mu \circ h^{4}\right| \Sigma_{A_{2}} \leqq 0$ and $\nu\left(A_{2}\right)<0$ or else $\left(^{*}\right)$ holds. Next suppose $\left(^{*}\right)$ holds. Since $\xi\left(A_{1}\right) \neq 0$ from the representation of $\xi$ it is verified that $\mu \circ h^{\Delta}\left(A_{1}\right) \neq 0$. Hence from $\left(^{*}\right)$ it follows that $\xi_{F}\left(A_{1}\right) \neq 0$. Thus there is a real number $\beta>0$ such that $\left(\beta \mu \circ h^{4} \mid \Sigma_{A_{1}}-\xi_{F}\right)\left(A_{1}\right)>0$. Hence from Jordan decomposition theorem it follows that there is a $\mathscr{B}$-measurable set $A_{2} \subset A_{1}$ satisfying the property (**). Thus from the preceding remarks we can choose the set $A$ itself to have the properties

(1) there exists $\alpha>0$ such that $f \chi_{h^{\Delta}(A)}>\alpha \chi_{h^{\Delta}(A)}$,

(2) there exists a $\gamma>0$ such that for all $B \in \mathscr{B}, B \subset A, \xi_{F}(B) \leqq$ $\gamma \mu \circ h^{\Delta}(B)$.

Now choose a pairwise disjoint sequence of $\mathscr{B}$-measurable sets $\left\{A_{i}\right\}_{i \geqq 1}$, $A_{i} \subset A, \xi\left(A_{i}\right) \neq 0$. Since $\xi<<\xi_{F}, \xi_{F}\left(A_{i}\right)>0$. We complete now the proof of non-existence of Radon-Nikodym derivative of $\xi$ with respect to $\xi_{F}$ by discussing the two cases $1 \leqq p<\infty, p=\infty$ separately. If $1 \leqq p<\infty$ with the sequence $\left\{A_{i}\right\}_{i \geqq 1}$ as chosen above consider the inequality, for $i \neq j$, $(i, j$, sufficiently large $)$,

$$
\begin{aligned}
\left\|\frac{f \chi_{h^{\lrcorner}\left(A_{i}\right)}}{\xi_{F}\left(A_{i}\right)}-\frac{f \chi_{h^{\Delta}\left(A_{j}\right)}}{\xi_{F}\left(A_{j}\right)}\right\|^{p} & =\left\|\frac{\xi_{F}\left(A_{j}\right) f \chi_{h{ }^{\Delta}\left(A_{i}\right)}-\xi_{F}\left(A_{i}\right) f \chi_{h^{\Delta}\left(A_{j}\right)}}{\xi_{F}\left(A_{i}\right) \xi_{F}\left(A_{j}\right)}\right\|^{p} \\
& \geqq \frac{\int\left|f \chi_{h^{\Delta}\left(A_{i}\right)}\right|^{p} d \mu}{\xi_{F}\left(A_{i}\right)} \geqq \frac{\alpha^{p} \mu h^{\Delta}\left(A_{i}\right)}{\gamma \mu \circ h^{\Delta}\left(A_{i}\right)}=\frac{\alpha^{p}}{\gamma} .
\end{aligned}
$$

Thus the sequence $\left\{f \chi_{h^{4}\left(A_{i}\right)} / \xi_{F}\left(A_{i}\right)\right\}_{i \geqq 1}$ does not admit a convergent subsequence. Hence it follows from Theorem 1, Rieffel [9] that $\xi_{F}$ does not admit a Radon-Nikodym derivative with respect to $\xi$. If $p=\infty$ it is verified that

$$
\left\|\frac{f \chi_{h^{\Delta}\left(A_{i}\right)}}{\xi_{F}\left(A_{i}\right)}-\frac{f \chi_{h^{4}\left(A_{j}\right)}}{\xi_{F}\left(A_{j}\right)}\right\| \geqq\left\|\frac{f \chi_{h}{ }_{\left(A_{i}\right)}}{\xi_{F}\left(A_{i}\right)}\right\| \geqq \frac{\alpha}{\xi_{F}(A)},
$$

for $i, j$ sufficiently large. Once again applying Rieffel's theorem, the proof of the theorem is completed for the case $p=\infty$.

REMARK 1. We note from the preceding theorem and Theorem 
1 in [9] that $\xi$ admits a Radon-Nikodym derivative with respect to $\xi_{F}$ iff

(1) $\xi<<\xi_{F}$,

(2) $|\xi|$, the variation of $\xi$, is a finite measure and

(3) $(T, \mathscr{B},|\xi|)$ is purely atomic.

REMARK 2. In $\S 4$, Masani [6] obtained sufficient conditions for the non-existence of Radon-Nikodym derivative of a c.a.l.o.s. measure $\xi$ taking values in a Hilbert space $\mathfrak{X}$ with respect to the measure $\xi_{F}(\cdot)=\|\xi(\cdot)\|^{2}$ and $F(x)=\|x\|^{2}$. The following analogue of Masani's theorem for c.a.l.o.s. measures $\xi$ is an immediate consequence of the preceding theorem.

CoRollary. Let $\xi: \mathscr{B} \rightarrow \mathscr{H}$ be a c.a.l.o.s. measure, where $\mathscr{H}$ is the Hilbert space $L_{2}(\Omega, \Sigma, \mu)$. Let $F: \mathscr{H} \rightarrow R$ be the additive functional defined by $F(x)=\|x\|^{2}$. Then $\xi$ admits a Radon-Nikodym derivative w.r.t. $\xi_{F}$ iff (1) $|\xi|$ is a finite measure and (2) $(T, \mathscr{B},|\xi|)$ is purely atomic.

6. In this section we apply the results obtained in the preceding sections to a probabilistic problem concerning independently scattered random measures.

Let $\mathfrak{M}(\pi, \mathscr{P}, P)$ be a complete metric linear space (topology determined by convergence in probability) of all random variables on a probability space $(\pi, \mathscr{P}, P)$. An independently scattered random measure on the Borel subsets $\mathscr{B}$ on the unit interval $T$ is a mapping $M: \mathscr{B} \rightarrow \mathfrak{M}$ enjoying the following properties.

$\left.1^{+}\right)$for every sequence $\left\{E_{i}\right\}$ of pairwise disjoint Borel sets

$$
M\left(\bigcup_{i=1}^{\infty} E_{i}\right)=\sum_{i=1}^{\infty} M\left(E_{i}\right)
$$

where the series converges with probability 1.

${ }^{(+)}$for every sequence $E_{1}, \cdots, E_{n}$ of disjoint Borel sets the random variables $M\left(E_{1}\right), \cdots, M\left(E_{n}\right)$ are independent. For the theory of such measures, the reader is referred to [12] and references given therein. The measure $M$ is said to be non-atomic if $M(\{a\})=0$ P.a.e. for every one point set $\{a\}$. Let $[M]$ denote the closed subspace spanned in $M$ by range of $M$. The definition and the properties of the integral of real functions on $T$ with respect to $M$ may be found in [12]. $L_{1}(M)$ denotes the space of $M$-integrable real-valued functions on $T$.

THEOREM 3. Let $M$ be a non-atomic and non-Gaussian independently scattered $\mathfrak{M}$-valued random measure. If $N: \mathscr{B} \rightarrow[M]$ is an 
independently scattered random measure, then there exists a Borel measurable mapping $\tau: T \rightarrow T$ and a function $f \in L_{1}(M)$ such that the measure $N$ has the following representation

$$
N(A)=\int_{\tau^{-1}(A)} f(t) M(d t), \quad A \in \mathscr{B} .
$$

Proof. For every Borel set $A, N(A) \in[M]$. Hence from the representation theorem in $\S 2$ of [12], there exists an $f_{A} \in L_{1}(M)$ such that

$$
N(A)=\int_{T} f_{A}(t) M(d t) .
$$

Since the mapping $N(A) \rightarrow f_{A}$ is a continuous linear mapping, the set function $\mathscr{B} \ni A \rightarrow f_{A}$ is a measure with values in the complete metric space $L_{1}(M)$. If $A$ and $B$ are disjoint Borel sets then $N(A)$ and $N(B)$ independent and from Theorem 2.1 in [12] $f_{A}$ and $f_{B}$ are orthogonal in the sense of $\S 1$. Thus the iet function $\mathscr{B} \ni A \rightarrow f_{A}$ is a c.a.l.o.s. $L_{1}(M)$ valued measure. It follows from Proposition 3 in $\S 4$ that

$$
f_{A}=f \chi_{\tau}^{-1(A)}
$$

for some $f \in L_{1}(M)$ and a Borel measurable mapping $\tau: T \rightarrow T$. This completes the proof of the theorem.

REMARK. Under the hypothesis of the preceding theorem, it follows from the theorem that $M<<N \circ \tau$, where $\tau: T \rightarrow T$ is a Borel measurable mapping.

In conclusion, it might be mentioned that we are not aware of any immediate applications of Theorem 3 in Stochastic processes, but the theorem itself seems to us to be surprising and of intrinsic interest.

\section{REFERENCES}

1. N. Dinculeanu, Vector Measures, Pergamon Press, 1967.

2. P. R. Halmos, Measure Theory, Van Nostrand, 1950.

3. A. and C. Ionescu-Tulcea, Topics in the Theory of Lifting, Springer-Verlag, 1969.

4. R. C. James, Orthogonality and Linear Functionals in Normed Linear Spaces, Trans. Amer. Math. Soc., 61 (1947), 261-292.

5. A. N. Kolmogorov, Stationary Sequences in Hilbert Spaces, (in Russian), Bulletin of M. G. U. 2 (1941), 1-40.

6. P. Masani, Orthogonally Scattered Measures, Advances in Math., 2 (1968), 61-117.

7. - Quasi-isometric Measures and Their Applications, Bull. Amer. Math. Soc., 76 (1970), 427-528.

8. W. Matuszewska and W. Orlicz, Note on Modular Spaces, IX, Bull. Polon. Ac. Sci., 16 (1968), 801-808.

9. M. A. Rieffel, The Radon-Nikodym Theorem for the Bochner Integral, Trans. Amer. Math. Soc., 131 (1968), 466-487.

10. R. Sikorski, Boolean Algebras, Springer-Verlag, 1964. 
11. K. Sundaresan, Orthogonality and Nonlinear Functionals on Banach Spaces, Report 71-22, Carnegie-Mellon University.

12. K. Urbanik, Random Measures and Harmonizable Sequences, Studia Math., 31 (1968), 61-88.

13. N. Wiener, Differential Space, J. Math. and Phys., 2 (1923), 131-174.

14. K. Yosida, Functional Analysis, Springer-Verlag, 1965.

Received July 13, 1971 and in revised form December 15, 1971. The research work of the first author was in part supported by a Scaife Faculty Grant administered by Carnegie-Mellon University. The research of the second author was in part supported by NSF Grant QU-9056 while on leave from Wroclaw University and the Institute of Mathematics of the Polish Academy of Sciences, Poland. 



\section{PACIFIC JOURNAL OF MATHEMATICS}

\section{EDITORS}

\author{
H. SAMELSON \\ Stanford University \\ Stanford, California 94305 \\ C. R. HOBBY \\ University of Washington \\ Seattle, Washington 98105
}

\section{J. DugundjI}

Department of Mathematics University of Southern California Los Angeles, California 90007

\section{RICHARD ARENS}

University of California

Los Angeles, California 90024

\section{ASSOCIATE EDITORS}
E. F. BECKENBACH
B. H. NeumanN
F. WOLF
K. YosHIDA

\section{SUPPORTING INSTITUTIONS}

\author{
UNIVERSITY OF BRITISH COLUMBIA \\ CALIFORNIA INSTITUTE OF TECHNOLOGY \\ UNIVERSITY OF CALIFORNIA \\ MONTANA STATE UNIVERSITY \\ UNIVERSITY OF NEVADA \\ NEW MEXICO STATE UNIVERSITY \\ OREGON STATE UNIVERSITY \\ UNIVERSITY OF OREGON \\ OSAKA UNIVERSITY
}

\author{
UNIVERSITY OF SOUTHERN CALIFORNIA \\ STANFORD UNIVERSITY \\ UNIVERSITY OF TOKYO \\ UNIVERSITY OF UTAH \\ WASHINGTON STATE UNIVERSITY \\ UNIVERSITY OF WASHINGTON \\ ${ }^{*} \stackrel{*}{*}{ }^{*}$
AMERICAN MATHEMATICAL SOCIETY
NAVAL WEAPONS CENTER
}

The Supporting Institutions listed above contribute to the cost of publication of this Journal, but they are not owners or publishers and have no responsibility for its content or policies.

Mathematical papers intended for publication in the Pacific Journal of Mathematics should be in typed form or offset-reproduced, (not dittoed), double spaced with large margins. Underline Greek letters in red, German in green, and script in blue. The first paragraph or two must be capable of being used separately as a synopsis of the entire paper. The editorial "we" must not be used in the synopsis, and items of the bibliography should not be cited there unless absolutely necessary, in which case they must be identified by author and Journal, rather than by item number. Manuscripts, in duplicate if possible, may be sent to any one of the four editors. Please classify according to the scheme of Math. Rev. Index to Vol. 39. All other communications to the editors should be addressed to the managing editor, Richard Arens, University of California, Los Angeles, California, 90024.

50 reprints are provided free for each article; additional copies may be obtained at cost in multiples of 50 .

The Pacific Journal of Mathematics is published monthly. Effective with Volume 16 the price per volume (3 numbers) is $\$ 8.00$; single issues, $\$ 3.00$. Special price for current issues to individual faculty members of supporting institutions and to individual members of the American Mathematical Society: $\$ 4.00$ per volume; single issues $\$ 1.50$. Back numbers are available.

Subscriptions, orders for back numbers, and changes of address should be sent to Pacific Journal of Mathematics, 103 Highland Boulevard, Berke'ey, California, 94708.

PUBLISHED BY PACIFIC JOURNAL OF MATHEMATICS, A NON-PROFIT CORPORATION

Printed at Kokusai Bunken Insatsusha (International Academic Printing Co., Ltd.), 270, 3-chome Totsuka-cho, Shinjuku-ku, Tokyo 160, Japan. 


\section{Pacific Journal of Mathematics}

\section{Vol. 43, No. $3 \quad$ May, 1972}

Max K. Agoston, An obstruction to finding a fixed point free map on a manifold.... 543

Nadim A. Assad and William A. Kirk, Fixed point theorems for set-valued mappings

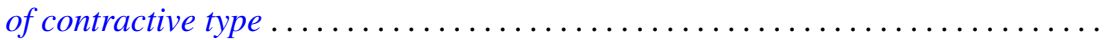

John Winston Bunce, Characterizations of amenable and strongly amenable

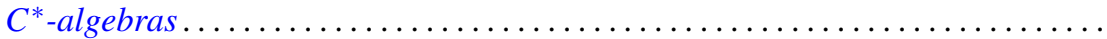

Erik Maurice Ellentuck and Alfred Berry Manaster, The decidability of a class of

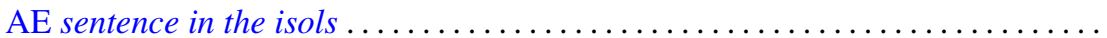

U. Haussmann, The inversion theorem and Plancherel's theorem in a Banach

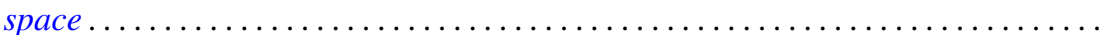

Peter Lawrence Falb and U. Haussmann, Bochner's theorem in infinite dimensions.

Peter Fletcher and William Lindgren, Quasi-uniformities with a transitive base ..... Dennis Garbanati and Robert Charles Thompson, Classes of unimodular abelian

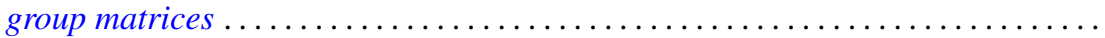

Kenneth Hardy and R. Grant Woods, On c-realcompact spaces and locally bounded normal functions

Manfred Knebusch, Alex I. Rosenberg and Roger P. Ware, Grothendieck and Witt rings of hermitian forms over Dedekind rings .......................

George M. Lewis, Cut loci of points at infinity.

Jerome Irving Malitz and William Nelson Reinhardt, A complete countable $L_{\omega_{1}}^{Q}$ theory with maximal models of many cardinalities . . . . . . . . . . . . . . . . .

Wilfred Dennis Pepe and William P. Ziemer, Slices, multiplicity, and Lebesgue

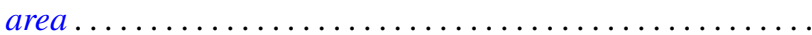

Keith Pierce, Amalgamating abelian ordered groups . .

Stephen James Pride, Residual properties of free groups . . . . . . . . . . . . . 725

Roy Martin Rakestraw, The convex cone of n-monotone functions .

T. Schwartzbauer, Entropy and approximation of measure preserving transformations .

Peter F. Stebe, Invariant functions of an iterative process for maximization of a polynomial...

Kondagunta Sundaresan and Wojbor Woyczynski, L-orthogonally scattered

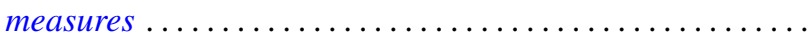

Kyle David Wallace, $C_{\lambda}$-groups and $\lambda$-basic subgroups $\ldots \ldots \ldots$

Barnet Mordecai Weinstock, Approximation by holomorphic functions on certain

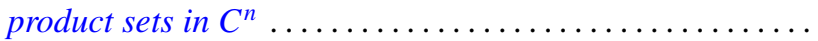

Donald Steven Passman, Corrections to: "Isomorphic groups and group rings”.

Don David Porter, Correction to: "Symplectic bordism, Stiefel-Whitney numbers, and a Novikov resolution"

John Ben Butler, Jr., Correction to: “Almost smooth perturbations of self-adjoint operators".

Constantine G. Lascarides, Correction to: "A study of certain sequence spaces of Maddox and a generalization of a theorem of Iyer" ...... ...

George A. Elliott, Correction to: "An extension of some results of takesaki in the reduction theory of von neumann algebras" ......................... 